ELECTRONIC RESEARCH ANNOUNCEMENTS OF THE AMERICAN MATHEMATICAL SOCIETY

Volume 10, Pages 135-141 (December 10, 2004)

S $1079-6762(04) 00138-6$

\title{
COMPACTNESS AND GLOBAL ESTIMATES FOR THE GEOMETRIC PANEITZ EQUATION IN HIGH DIMENSIONS
}

\author{
EMMANUEL HEBEY AND FRÉDÉRIC ROBERT \\ (Communicated by Tobias Colding)
}

\begin{abstract}
Given $(M, g)$, a smooth compact Riemannian manifold of dimension $n \geq 5$, we investigate compactness for the fourth order geometric equation $P_{g} u=u^{2^{\sharp}-1}$, where $P_{g}$ is the Paneitz operator, and $2^{\sharp}=2 n /(n-4)$ is critical from the Sobolev viewpoint. We prove that the equation is compact when the Paneitz operator is of strong positive type.
\end{abstract}

In 1983, Paneitz [9] introduced a conformally fourth order operator defined on 4-dimensional Riemannian manifolds. Branson [3] generalized the definition to $n$-dimensional Riemannian manifolds, $n \geq 5$. While the conformal Laplacian is associated to the scalar curvature, the geometric Paneitz-Branson operator is associated to a notion of $Q$-curvature. The $Q$-curvature in dimension 4 , and for conformally flat manifolds, is the integrand in the Gauss-Bonnet formula for the Euler characteristic. In this article we let $(M, g)$ be a smooth compact conformally flat Riemannian $n$-manifold, $n \geq 5$, and consider the geometric Paneitz equation

$$
P_{g} u=u^{2^{\sharp}-1},
$$

where $P_{g}$ is the Paneitz operator in dimension $n \geq 5, u$ is required to be positive, and $2^{\sharp}=\frac{2 n}{n-4}$ is the critical exponent for the Sobolev embedding. The Paneitz operator in dimension $n \geq 5$ reads as

$$
P_{g} u=\Delta_{g}^{2} u-\operatorname{div}_{g}\left(A_{g} d u\right)+\frac{n-4}{2} Q_{g} u,
$$

where $\Delta_{g}=-\operatorname{div}_{g} \nabla$ is the Laplace-Beltrami operator, $Q_{g}$ is the $Q$-curvature of $g$, $A_{g}$ is the smooth symmetrical $(2,0)$-tensor field given by

$$
A_{g}=\frac{(n-2)^{2}+4}{2(n-1)(n-2)} S_{g} g-\frac{4}{n-2} R c_{g},
$$

and $R c_{g}$ and $S_{g}$ are respectively the Ricci curvature and scalar curvature of $g$. The Paneitz operator is conformally invariant in the sense that if $\tilde{g}=u^{4 /(n-4)} g$ is conformal to $g$, then $P_{\tilde{g}}(f)=u^{s} P_{g}(u f)$ for all $f \in C^{\infty}(M)$, where $s=1-2^{\sharp}$. From the viewpoint of conformal geometry, equation (0.1) turns out to be the natural fourth order analogue of the second order Yamabe equation. We refer to Chang [4] and Chang and Yang [5] for more details on the above definitions.

Received by the editors October 7, 2004.

2000 Mathematics Subject Classification. Primary: 58E30, 58J05.

Key words and phrases. Blow-up behavior, compactness, Paneitz operator.

(C)2004 American Mathematical Society Reverts to public domain 28 years from publication 
In what follows we let $H_{2}^{2}(M)$ be the Sobolev space consisting of functions in $L^{2}(M)$ with two derivatives in $L^{2}$. As shown in Hebey and Robert [7], up to passing to a subsequence, bounded sequences $\left(u_{\alpha}\right)$ in $H_{2}^{2}(M)$ of nonnegative solutions of (0.1) Split into the sum of a nonnegative solution $u^{0}$, namely the weak limit of the $u_{\alpha}$, a finite sum of $k$ bubbles $\left(B_{\alpha}^{i}\right)$, obtained by rescaling positive solutions of the Euclidean equation $\Delta^{2} u=u^{2^{\sharp}-1}$, and a remainder $R_{\alpha}$ which converges strongly to zero in $H_{2}^{2}(M)$ as $\alpha \rightarrow+\infty$. This splitting provides exact asymptotics for the $u_{\alpha}$ in the Sobolev setting. Following standard terminology, we say that equation (0.1) is compact if for any bounded sequence $\left(u_{\alpha}\right)$ in $H_{2}^{2}(M)$ of nonnegative solutions of (0.1), we necessarily have that $k=0$ in such decompositions. Regularity theory (see for instance Esposito-Robert [6]) holds for (0.1). Then, thanks to Agmon-Douglis-Nirenberg-type estimates [1, 2], and estimates like the ones developed in Hebey, Robert, and Wen [8], an equivalent definition for compactness is that bounded sequences in $H_{2}^{2}(M)$ of nonnegative solutions of (0.1) are actually bounded in $C^{4, \theta}(M)$. A major stress in studying compactness is to understand large solutions. Namely, solutions with large energies which, in studying their possible blow-up, involve multi-bubbles which may interact with each other on the pointwise level.

Compactness for fourth order equations like 0.1 was recently studied in Hebey, Robert, and Wen [8]. It was shown in [8] that equations like [0.1) are compact as long as they are not close to the geometric equation (0.1). In this note we investigate compactness for the geometric equation (0.1) and show how the analysis developed in 8 . provides an answer to the question of whether (0.1) is compact or not. The result we get is the fourth order analogue of the result proved in Schoen [10], where the second order Yamabe equation was investigated. As in Hebey, Robert, and Wen [8, and also Schoen [10], we assume in what follows that $(M, g)$ is conformally flat (and hence, since $n \geq 5$, that the Weyl tensor of $g$ is zero). We let $G_{g}$ be the Green's function of $P_{g}$. The Green's function is unique if $P_{g}$ is positive. By conformal invariance of the Paneitz operator, if $\tilde{g}=u^{4 /(n-4)} g$ is a conformal metric to $g$, then

$$
G_{\tilde{g}}(x, y)=\frac{G_{g}(x, y)}{u(x) u(y)} .
$$

It is known that if $\tilde{g}$ is a flat metric around some $x_{0} \in M$, then

$$
G_{\tilde{g}}\left(x_{0}, x\right)=\frac{\lambda_{n}}{d_{\tilde{g}}\left(x_{0}, x\right)^{n-4}}+\mu_{\tilde{g}}\left(x_{0}, x\right),
$$

where $\lambda_{n}^{-1}=2(n-2)(n-4) \omega_{n-1}, \omega_{n-1}$ is the volume of the unit $(n-1)$-sphere, and the function $x \rightarrow \mu_{\tilde{g}}\left(x_{0}, x\right)$ is smooth on $M$. Combining the above two equations, noting that conformal changes of metrics which leave a metric flat around one point come from conformal diffeomorphisms of the Euclidean space, we easily get (as in Schoen and Yau [11] for the conformal Laplacian) that if $g$ and $\tilde{g}=u^{4 /(n-4)} g$ are conformal metrics, both being flat around $x_{0}$, then

$$
\mu_{\tilde{g}}\left(x_{0}, x_{0}\right)=\frac{\mu_{g}\left(x_{0}, x_{0}\right)}{u\left(x_{0}\right)^{2}} .
$$

In particular, by (0.4), the sign of $\mu_{g}\left(x_{0}\right)=\mu_{g}\left(x_{0}, x_{0}\right)$ does not depend on the choice of the metric in $[g]_{x_{0}}$, where $[g]_{x_{0}}$ stands for the set of conformal metrics to $g$ which are flat around $x_{0}$. 
In what follows we say that $P_{g}$ is of strong positive type if $P_{g}$ is positive, $G_{g}$ is positive, and for any $x \in M$ there exists $\tilde{g} \in[g]_{x}$ such that $\mu_{\tilde{g}}(x)>0$. For example, the Paneitz operator on quotients of the unit sphere is of strong positive type. Positivity of the Paneitz operator was studied in Xu and Yang [12]. The main result of this note is:

Theorem 0.1. The geometric equation (0.1) is compact on compact conformally flat manifolds of dimensions $n \geq 5$ with Paneitz operator of strong positive type.

Let $(M, g)$ be a smooth compact conformally flat manifold of dimension $n \geq 5$ with positive Paneitz operator $P_{g}$, and positive Green's function $G_{g}$. Given $S \subset M$, let $[g]_{S}$ be the set of conformal metrics to $g$ which are flat in a neighborhood of $S$. We prove Theorem 0.1 by proving that if $\left(u_{\alpha}\right)$ is a bounded sequence in $H_{2}^{2}(M)$ of nonnegative solutions of (0.1) which blows up with geometric blow-up points $S=\left\{x_{1}, \ldots, x_{N}\right\}$, then $u_{\alpha} \rightarrow 0$ in $H_{2}^{2}(M)$ as $\alpha \rightarrow+\infty$, and for any $\tilde{g} \in[g]_{S}$, there exist $\lambda_{i, j}>0$ such that for any $i=1, \ldots, N$,

$$
\mu_{\tilde{g}}\left(x_{i}\right)+\sum_{j \neq i} \lambda_{i, j} G_{\tilde{g}}\left(x_{i}, x_{j}\right)=0 .
$$

Theorem 0.1 clearly follows from (0.5). We prove equation (0.5) in the rest of this note, using the material proved in Hebey, Robert, and Wen [8].

\section{Proof of the Result}

In what follows we prove (0.5), and thus Theorem 0.1 . For that purpose we let $\left(u_{\alpha}\right)$ be a bounded sequence in $H_{2}^{2}(M)$ of nonnegative nontrivial solutions of (0.1). Since the Green's function $G_{g}$ of $P_{g}$ is positive, the $u_{\alpha}$ are positive. In the sequel, everything is up to a subsequence. We know from Hebey and Robert [7] that there exist $k \in \mathbb{N}$, a nonnegative solution $u^{0} \geq 0$ of (0.1), and $k$ bubbles $\left(B_{\alpha}^{i}\right)$, $i=1, \ldots, k$, such that

$$
u_{\alpha}=u^{0}+\sum_{i=1}^{k} B_{\alpha}^{i}+R_{\alpha},
$$

where $R_{\alpha} \rightarrow 0$ in $H_{2}^{2}(M)$ as $\alpha \rightarrow+\infty$. By contradiction, we assume that $k \geq 1$, and let $S=\left\{x_{1}, \ldots, x_{N}\right\}$ be the geometric blow-up point set consisting of the limits of the centers of the bubbles $\left(B_{\alpha}^{i}\right)$. Since bubbles may accumulate one on another (there are such examples for equations like (10.1); we refer to Hebey, Robert, and Wen [8]), $N$ might be less than $k$. By conformal invariance we may assume that $g=\tilde{g}$ is flat around the points in $S$. Then the geometric Paneitz equation (0.1) reduces to $\Delta_{g}^{2} u_{\alpha}=u_{\alpha}^{2^{\sharp}-1}$ around the points in $S$.

As a preliminary step in the proof of (0.5), we come back to the estimates proved in Hebey, Robert, and Wen [8] and explain why they are still valid in the present context. A rough argument would be that blow-up phenomena are local in nature, while the Paneitz operator on conformally flat manifolds is locally, up to conformal changes of the metric, like the Paneitz operator on the sphere (and hence with positive constant coefficients as in [8]). More details follow. First we note that the standard procedure to get rescaling invariant pointwise estimates, as developed in 
[8] for fourth order operators, together with Agmon-Douglis-Nirenberg-type estimates for fourth order operators, give that there exists $C>0$ such that,

$$
\left(\min _{1 \leq i \leq k} d_{g}\left(x_{\alpha}^{i}, x\right)\right)^{\frac{n-4}{2}}\left|u_{\alpha}(x)-u^{0}(x)\right| \leq C
$$

for all $\alpha$ and all $x$, where $u^{0}$, the weak limit of the $u_{\alpha}$, is as in (1.1), and where the $x_{\alpha}^{i}, i=1, \ldots, k$, are the centers of the bubbles $\left(B_{\alpha}^{i}\right)$ in (1.1). In particular, we get with (1.2) that $u_{\alpha} \rightarrow u^{0}$ in $C_{l o c}^{4}(M \backslash S)$ as $\alpha \rightarrow+\infty$. In addition to (1.2), if $\Phi_{\alpha}(x)$ stands for the left hand side in (1.2), we also have that

$$
\lim _{R \rightarrow+\infty} \lim _{\alpha \rightarrow+\infty} \sup _{x \in M \backslash \Omega_{\alpha}(R)} \Phi_{\alpha}(x)^{\frac{n-4}{2}}=0,
$$

where, for $R>0, \Omega_{\alpha}(R)$ is given by $\Omega_{\alpha}(R)=\bigcup_{i=1}^{k} B_{x_{\alpha}^{i}}\left(R \mu_{\alpha}^{i}\right)$, and the $\mu_{\alpha}^{i}$ are the weights of the bubbles $\left(B_{\alpha}^{i}\right)$ in (1.2). Going on with the estimates in Hebey, Robert, and Wen [8] we may assume, up to renumbering, and up to passing to a subsequence, that $\left(B_{\alpha}^{1}\right)$ is the bubble in (1.1) with the largest weight. Then we let the $x_{\alpha}$ and $\mu_{\alpha}$ be such that $x_{\alpha}=x_{\alpha}^{1}$ and $\mu_{\alpha}=\mu_{\alpha}^{1}$ for all $\alpha$. A preliminary remark is that the global splitting estimate $\left\|u_{\alpha}\right\|_{p_{1}, p_{2}, \mu_{\alpha}^{-1}} \leq C$ in $[8$ ] easily follows from the positivity of the Green's function with only slight modifications of the arguments in 8]. The arguments in [8] used the decomposition of the fourth order operator into the product of two second order operators. We may use instead Agmon-DouglisNirenberg-type estimates and note that the positivity of the Green's function $G_{g}$ implies that $P_{g}$ satisfies the comparison principle. As an independent easy remark, since $\Delta_{g}\left(\Delta_{g} u_{\alpha}\right) \geq 0$ around the points in $S$, there exists $C>0$ such that $\Delta_{g} u_{\alpha} \geq$ $-C$ in $M$, for all $\alpha$. Then, with such an estimate, and the analysis developed in 8, we easily get that the integral and asymptotic estimates in Hebey, Robert, and Wen [8] follow from (1.1)-(1.3). In several places the computations in [8] simplify because of the simple nature of the geometric equation around the points in $S$. By the asymptotic estimates, if we let $\tilde{u}_{\alpha}$ be the rescaled function obtained from $u_{\alpha}$ by $\tilde{u}_{\alpha}(x)=u_{\alpha}\left(\exp _{x_{\alpha}}\left(\sqrt{\mu_{\alpha}} x\right)\right)$, we get that there exist $\delta>0, A>0$, and a biharmonic function $\varphi \in C^{4}\left(B_{0}(2 \delta)\right)$ such that, up to a subsequence,

$$
\tilde{u}_{\alpha}(x) \rightarrow \frac{A}{|x|^{n-4}}+\varphi(x)
$$

in $C_{l o c}^{3}\left(B_{0}(2 \delta) \backslash\{0\}\right)$ as $\alpha \rightarrow+\infty$. Moreover, since we assumed that $G_{g}>0$, so that either $u^{0} \equiv 0$ or $u^{0}>0$ everywhere, we also have the important information that $\varphi$ is positive in $B_{0}(2 \delta)$ if $u^{0} \not \equiv 0$.

As an important step in the proof of (0.5), we claim that thanks to the asymptotics (1.4), and thanks to the property that $\varphi$ is positive if $u^{0}$ is nonzero, we necessarily have that $u^{0} \equiv 0$ when the $u_{\alpha}$ blow up. In order to do this, we use the Pohozaev [Pokhozhaev] identity as in [8], and conformal invariance. The Pohozaev identity for fourth order equations reads as

$$
\begin{aligned}
\int_{\Omega}\left(x^{k} \partial_{k} u\right) \Delta^{2} u d x+\frac{n-4}{2} \int_{\Omega} u \Delta^{2} u d x \\
=\frac{n-4}{2} \int_{\partial \Omega}\left(-u \frac{\partial \Delta u}{\partial \nu}+\frac{\partial u}{\partial \nu} \Delta u\right) d \sigma \\
\quad+\int_{\partial \Omega}\left(\frac{1}{2}(x, \nu)(\Delta u)^{2}-(x, \nabla u) \frac{\partial \Delta u}{\partial \nu}+\frac{\partial(x, \nabla u)}{\partial \nu} \Delta u\right) d \sigma
\end{aligned}
$$


for all smooth bounded domains $\Omega$ in $\mathbb{R}^{n}$ and all $u \in C^{4}(\bar{\Omega})$, where $\Delta$ is the Euclidean Laplacian, $\nu$ is the outward unit normal of $\partial \Omega$, and $d \sigma$ is the Euclidean volume element on $\partial \Omega$. We apply the Pohozaev identity (1.5) to the $u_{\alpha}$ in the ball $\Omega=B_{0}\left(\delta \sqrt{\mu_{\alpha}}\right)$. In the process we assimilate $x_{\alpha}$ and 0 (thanks to the exponential map at $x_{\alpha}$ ), and regard $u_{\alpha}$ as a function in the Euclidean space. Noting that

$$
\int_{B_{\alpha}}\left(x^{k} \partial_{k} u_{\alpha}\right) \Delta^{2} u_{\alpha} d x+\frac{n-4}{2} \int_{B_{\alpha}} u_{\alpha} \Delta^{2} u_{\alpha} d x=O\left(\int_{\partial B_{\alpha}} u_{\alpha}^{2^{\sharp}} d \sigma\right),
$$

where $B_{\alpha}=B_{0}\left(\delta \sqrt{\mu_{\alpha}}\right)$, and that $\int_{\partial B_{\alpha}} u_{\alpha}^{2^{\sharp}} d \sigma=o\left(\mu_{\alpha}^{(n-4) / 2}\right)$ by (1.4), we get with (1.4), the Pohozaev identity, and the computations developed in [8], that

$$
(n-2)(n-4)^{2} \omega_{n-1} A \varphi(0) \mu_{\alpha^{\frac{n-4}{2}}}+o\left(\mu_{\alpha^{\frac{n-4}{2}}}\right)=0,
$$

where $\omega_{n-1}$ is the volume of the unit $(n-1)$-sphere, and $A$ and $\varphi$ are as in (1.4). In particular, $\varphi(0)=0$, and since $\varphi>0$ if $u^{0} \not \equiv 0$, this proves the above claim that we necessarily have that $u^{0} \equiv 0$ when the $u_{\alpha}$ blow up. With respect to the terminology in Hebey, Robert, and Wen [8], this amounts to saying that compactness reduces to pseudo-compactness for the geometric equation.

Going on with the proof of $(0.5)$, and now that we know that $u^{0} \equiv 0$, we need to add one important estimate to the estimates listed above, which we proved in [8]. We claim here that

$$
\lambda_{\alpha} u_{\alpha}(x) \rightarrow \sum_{i=1}^{N} \lambda_{i} G_{g}\left(x_{i}, x\right)
$$

in $C_{l o c}^{4}(M \backslash S)$ as $\alpha \rightarrow+\infty$, where $\lambda_{\alpha} \rightarrow+\infty$ as $\alpha \rightarrow+\infty, S=\left\{x_{1}, \ldots, x_{N}\right\}$ is the geometric blow-up point set of the $u_{\alpha}, G_{g}$ is the Green's function of $P_{g}$, and the $\lambda_{i} \geq 0, i=1, \ldots, N$, are such that $\sum_{i=1}^{N} \lambda_{i}=1$. In order to prove (1.6), we use the positivity of $P_{g}$ and $G_{g}$ as follows. By the positivity of $P_{g}$, the lowest eigenvalue $\lambda$ of $P_{g}$ is positive. If $\psi$ is an eigenfunction for $\lambda$, letting $P_{g} u=\left|P_{g} \psi\right|$, and writing that $P_{g} u \geq P_{g} \psi$ and $P_{g} u \geq-P_{g} \psi$, we get with the positivity of $G_{g}$ that $u \geq|\psi|$. Noting that $u>0$ since $P_{g} u=\lambda|\psi|$ and $G_{g}>0$, plugging $u$ into the Rayleigh characterization of $\lambda$, it follows that either $\psi<0$ or $\psi>0$. Without loss of generality we may assume that $\psi>0$. Then the conformal metric $\tilde{g}=\psi^{4 /(n-4)} g$ is such that $Q_{\tilde{g}}>0$, where $Q_{\tilde{g}}$ is the $Q$-curvature of $\tilde{g}$. By conformal invariance, $u=\psi^{-1} u_{\alpha}$ solves the equation $P_{\tilde{g}} u=u^{2^{\sharp}-1}$. Integrating over $M$, since $Q_{\tilde{g}}>0$, we get that there exists $C>0$ such that $\left\|u_{\alpha}\right\|_{L^{1}(M)} \leq C\left\|u_{\alpha}\right\|_{L^{s}(M)}^{s}$ where $s=2^{\sharp}-1$. By Agmon-Douglis-Nirenberg-type estimates, noting that (1.2) gives that the $u_{\alpha}$ are bounded in $C_{\text {loc }}^{0}(M \backslash S)$ as $\alpha \rightarrow+\infty$, we get that for any $p>1$, and any $\delta>0$, the $L^{\infty}$-norm of the $u_{\alpha}$ in sets like $M \backslash B_{\delta}$ is controled by the $L^{p_{-}}$ norm of the $u_{\alpha}$ in $M$, where $B_{\delta}$ is the union over the $x \in S$ of the geodesic balls $B_{x}(\delta)$. By the above estimate, using Hölder's inequality with $1 \leq p \leq 2^{\sharp}$, choosing $p>1$ close to 1 , and since $u_{\alpha} \rightarrow 0$ in $L^{q}(M)$ for $q<2^{\sharp}$, it easily follows that $\left\|u_{\alpha}\right\|_{L^{s}\left(M \backslash B_{\delta}\right)}=o\left(\left\|u_{\alpha}\right\|_{L^{s}(M)}\right)$ for all $\delta>0$, where $s$ is as above. In particular, the $\lambda_{i}$ given by

$$
\lambda_{i}=\lim _{\alpha \rightarrow+\infty} \frac{\int_{B_{x_{i}}(\delta)} u_{\alpha}^{2^{\sharp}-1} d v_{g}}{\int_{M} u_{\alpha}^{2^{\sharp}-1} d v_{g}}
$$


are nonnegative, independent of $\delta>0$ small, and such that $\sum \lambda_{i}=1$. In what follows we let $\lambda_{\alpha}=\left\|u_{\alpha}\right\|_{2^{\sharp}-1}^{1-2^{\sharp}}$. Then $\lambda_{\alpha} \rightarrow+\infty$ as $\alpha \rightarrow+\infty$, while we can write with the Green's representation formula that for $x \in M \backslash B_{\delta}$, and $0<\delta^{\prime} \ll \delta$,

$$
\begin{aligned}
u_{\alpha}(x) & =\int_{B_{\delta^{\prime}}} G_{g}(x, y) u_{\alpha}^{2^{\sharp}-1}(y) d v_{g}(y)+\int_{M \backslash B_{\delta^{\prime}}} G_{g}(x, y) u_{\alpha}^{2^{\sharp}-1}(y) d v_{g}(y) \\
& =\left(\sum_{i=1}^{N} \lambda_{i} G_{g}\left(x_{i}, x\right)+o_{\delta^{\prime}}(1)\right) \lambda_{\alpha}^{-1},
\end{aligned}
$$

where $\lim _{\delta^{\prime} \rightarrow 0} \lim _{\alpha \rightarrow+\infty} o_{\delta^{\prime}}(1)=0$. In particular, $\lambda_{\alpha} u_{\alpha}(x) \rightarrow \sum_{i=1}^{N} \lambda_{i} G_{g}\left(x_{i}, x\right)$ in $C_{l o c}^{0}(M \backslash S)$ as $\alpha \rightarrow+\infty$, an equation from which we easily get that (1.6) is true.

With (1.6) we can now end the proof of (0.5). If $f$ stands for the function on the right hand side of (1.6), then $\Delta^{2} f=0$ in a set like $\Omega=\bigcup_{i=1}^{N} B_{x_{i}}\left(\delta_{0}\right) \backslash S$, where $\delta_{0}>0$. We apply the Pohozaev identity (1.5) to the $u_{\alpha}$ in $B_{x_{i}}(\delta)$ for $\delta>0$ small and $i$ in $\{1, \ldots, N\}$. In the process we assimilate $x_{i}$ and 0 (thanks to the exponential map at $x_{i}$ ), and regard $u_{\alpha}$ as a function in the Euclidean space. Noting that

$$
\int_{B}\left(x^{k} \partial_{k} u_{\alpha}\right) \Delta^{2} u_{\alpha} d x+\frac{n-4}{2} \int_{B} u_{\alpha} \Delta^{2} u_{\alpha} d x=O\left(\int_{\partial B} u_{\alpha}^{2^{\sharp}} d \sigma\right),
$$

where $B=B_{0}(\delta)$, it follows from (1.6) and the Pohozaev identity that

$$
\begin{aligned}
& \frac{n-4}{2} \int_{\partial B_{0}(\delta)}\left(-f \frac{\partial \Delta f}{\partial \nu}+\frac{\partial f}{\partial \nu} \Delta f\right) d \sigma \\
& \quad+\int_{\partial B_{0}(\delta)}\left(\frac{1}{2}(x, \nu)(\Delta f)^{2}-(x, \nabla f) \frac{\partial \Delta f}{\partial \nu}+\frac{\partial(x, \nabla f)}{\partial \nu} \Delta f\right) d \sigma=0 .
\end{aligned}
$$

By (0.3), and (1.6), we can write that

$$
f(x)=\frac{\hat{\lambda}_{i}}{\left|x-x_{i}\right|^{n-4}}+R_{i}(x)
$$

for $x \neq x_{i}$ close to $x_{i}$, where $\lambda_{i}$ is as in (1.6), $\hat{\lambda}_{i}=\lambda_{n} \lambda_{i}, \lambda_{n}$ is as in (0.3), $R_{i}$ is smooth around $x_{i}$, and

$$
R_{i}\left(x_{i}\right)=\lambda_{i} \mu_{g}\left(x_{i}\right)+\sum_{j \neq i} \lambda_{j} G_{g}\left(x_{j}, x_{i}\right) .
$$

Plugging these equations into (1.7) and letting $\delta \rightarrow 0$, we get that $R_{i}\left(x_{i}\right)=0$. This equation holds for all $i=1, \ldots, N$, and we assumed that $G_{g}>0$. It follows that $\lambda_{i}>0$ for all $i$. This ends the proof of $(0.5)$ and of Theorem 0.1 .

As a remark, Theorem 0.1 still holds if we replace the critical exponent $2^{\sharp}$ in (0.1) by $2^{\sharp}-p_{\alpha}$, where $p_{\alpha} \geq 0$ is such that $p_{\alpha} \rightarrow 0$ as $\alpha \rightarrow+\infty$. In this case the left hand side in (0.5) is not zero anymore, but is nonpositive. Needless to say, compactness of the subcritical equations provides a minimizing solution of (0.1).

\section{REFERENCES}

[1] S. Agmon, A. Douglis, and L. Nirenberg, Estimates near the boundary for solutions of elliptic partial differential equations satisfying general boundary conditions. I, Comm. Pure Appl. Math. 12 (1959), 623-727. MR0125307 (23:A2610) 
[2] S. Agmon, A. Douglis, and L. Nirenberg, Estimates near the boundary for solutions of elliptic partial differential equations satisfying general boundary conditions. II, Comm. Pure Appl. Math. 17 (1964), 35-92. MR0162050 (28:5252)

[3] T.P. Branson, Group representations arising from Lorentz conformal geometry, J. Funct. Anal. 74 (1987), 199-291. MR0904819 (90b:22016)

[4] S.Y.A. Chang, On Paneitz operator-a fourth order differential operator in conformal geometry, Harmonic Analysis and Partial Differential Equations, Essays in honor of Alberto P. Calderon, Eds: M. Christ, C. Kenig and C. Sadorsky, Chicago Lectures in Mathematics, 1999, 127-150. MR1743859 (2001g:58059)

[5] S.Y.A. Chang and P.C. Yang, On a fourth order curvature invariant, Comp. Math. 237, Spectral Problems in Geometry and Arithmetic, Ed: T. Branson, AMS, 9-28, 1999. MR1710786 (2001b:58056)

[6] P. Esposito and F. Robert, Mountain pass critical points for Paneitz-Branson operators, Calc. Var. Partial Differential Equations 15 (2002), 493-517. MR.1942129(2005a:58054)

[7] E. Hebey and F. Robert, Coercivity and Struwe's compactness for Paneitz type operators with constant coefficients, Calc. Var. Partial Differential Equations 13 (2001), 491-517. MR:1867939 (2003m:58051)

[8] E. Hebey, F. Robert, and Y. Wen, Compactness and global estimates for a fourth order equation of critical Sobolev growth arising from conformal geometry, Preprint of the University of Nice, 697, 2004.

[9] S. Paneitz, A quartic conformally covariant differential operator for arbitrary pseudoRiemannian manifolds, Preprint, 1983.

[10] R. Schoen, Variational theory for the total scalar curvature functional for Riemannian metrics and related topics, Topics in Calculus of Variations (Montecatini Terme, 1987), Lecture Notes in Math. 1365, Springer-Verlag, Berlin, 1989, pp. 120-154. MR0994021 (90g:58023)

[11] R. Schoen and S.T. Yau, Conformally flat manifolds, Kleinian groups and scalar curvature, Invent. Math. 92 (1988), 47-71. MR0931204 (89c:58139)

[12] X. Xu and P. Yang, Positivity of Paneitz operators, Disc. Cont. Dynamical Systems 7 (2001), 329-342. MR1808405 (2002d:58043)

Université de Cergy-Pontoise, Département de Mathématiques, Site de Saint-Martin, 2 avenue Adolphe Chauvin, 95302 Cergy-Pontoise cedex, France

E-mail address: Emmanuel.Hebey@math.u-cergy.fr

Laboratoire J.A.Dieudonné, Université de Nice Sophia-Antipolis, Parc Valrose, 06108 Nice CEDEX 2, France

E-mail address: frobert@math.unice.fr 